\title{
A Geometric View of Signal Sensitivity Metrics in multi-echo fMRI
}

\author{
Thomas T. Liu ${ }^{\mathrm{a}, \mathrm{b}, *}$, Bochao Lic , Brice Fernandez ${ }^{\mathrm{d}}$, Suchandrima Banerjee ${ }^{\mathrm{e}}$ \\ ${ }^{a}$ Center for Functional MRI, University of California San Diego, 9500 Gilman Drive MC 0677, La Jolla, CA 92093 \\ ${ }^{b}$ Departments of Radiology, Psychiatry, and Bioengineering, University of California San Diego, 9500 Gilman Drive, La \\ Jolla, CA 92093 \\ ${ }^{c}$ Department of Biomedical Engineering, University of Southern California, Los Angeles, CA, USA \\ ${ }^{d}$ GE Healthcare, Buc, France \\ ${ }^{e}$ GE Healthcare, Menlo Park, CA, USA
}

\begin{abstract}
In multi-echo fMRI (ME-fMRI), two metrics have been widely used to measure the performance of various acquisition and analysis approaches. These are temporal SNR (tSNR) and differential contrast-to-noise ratio (dCNR). A key step in ME-fMRI is the weighted combination of the data from multiple echoes, and prior work has examined the dependence of tSNR and dCNR on the choice of weights. However, most studies have focused on only one of these two metrics, and the relationship between the two metrics has not been examined. In this work, we present a geometric view that offers greater insight into the relation between the two metrics and their weight dependence. We identify three major regimes: (1) a tSNR robust regime in which tSNR is robust to the weight selection with most weight variants achieving close to optimal performance, whereas dCNR shows a pronounced dependence on the weights with most variants achieving suboptimal performance; (2) a dCNR robust regime in which dCNR is robust to the weight selection with most weight variants achieving close to optimal performance, while tSNR exhibits a strong dependence on the weights with most variants achieving significantly lower than optimal performance; and (3) a within-type robust regime in which both tSNR and dCNR achieve nearly optimal performance when the form of the weights are variants of their respective optimal weights and exhibit a moderate decrease in performance for other weight variants. Insight into the behavior observed in the different regimes is gained by considering spherical representations of the weight dependence of the components used to form each metric.
\end{abstract}

Keywords: fMRI, multi-echo, CNR, tSNR

\footnotetext{
*Corresponding Author

Email address: ttliu@ucsd.edu (Thomas T. Liu)
} 


\section{Introduction}

In multi-echo functional magnetic resonance imaging (ME-fMRI), time series data are acquired at various echo times (TE) and then typically combined to form a composite time series, formed as the weighted sum of the data from the different echoes. The potential benefits of ME-fMRI include the ability to increase sensitivity to functional changes and the capacity to more effectively distinguish BOLD-related functional signal changes from non-BOLD contributions (Kundu et al., 2017; Cohen et al., 2021) .

In evaluating the performance of ME-fMRI methods, two measures of sensitivity have been typically used. These are (1) temporal signal-to-noise-ratio (tSNR) (Kundu et al., 2013; Puckett et al., 2018 Cohen et al., 2017, Heunis et al., 2021; Cohen et al., 2021) and (2) differential contrast-to-noise ratio (dCNR), also known as pseudo-temporal BOLD Sensitivity (ptBS) (Poser et al., 2006: Kettinger et al. 2016). In general, the contrast-to-noise ratio (CNR) of a functional time series is computed as the ratio of the functional signal change divided by the temporal standard deviation $\sigma$. For a single-echo acquisition, CNR can be expressed as the product of the normalized BOLD signal change multiplied by tSNR, defined as the temporal mean of the BOLD signal divided by $\sigma$ (Parrish et al. 2000; Krüger and Glover, 2001). Thus, for an expected functional BOLD signal change (which depends on factors such as echo time, magnetic field strength, experimental design, and physiology), the CNR of a single-echo acquisition is maximized when tSNR is maximized.

For a multi-echo acquisition, the computation of CNR must take into account both the dependence of the BOLD signal change on echo time (TE) (Posse et al., 1999; Poser et al., 2006, Gowland and Bowtell, 2007) and the weight assigned to each echo. This results in an expression where the CNR can be expressed as the product of the expected change $-\Delta R_{2}^{*}$ in effective transverse relaxation rate and dCNR, such that for a given change in the relaxation rate CNR is maximized when dCNR is maximized (see also Theory section below). To compute tSNR, the temporal mean of the composite time series is divided by its standard deviation. In the theoretical limiting case where all of the echo times are set equal to a single reference echo time (note that this is not done in practice), dCNR is equal to tSNR multiplied by the reference echo time (supporting details in Theory below). However, for an arbitrary choice of echo times, dCNR and tSNR are not necessarily proportional and the relation between CNR and tSNR is not straightforward.

Prior work has considered the weight dependence of both metrics. In a seminal paper, Posse et al. 
with matched-filter weighting under the assumption that the noise is uncorrelated with equal variances across echoes. This weighting scheme has been adopted by a number of subsequent studies and referred to by a variety of names, such as optimal combination (Kundu et al., 2013), BOLD-signal weighted (Poser et al. 2006, Kettinger et al. 2016), and $T_{2} *$-weighted (Heunis et al., 2021). Other weighting schemes have

also been explored, such as a flat weighting in which all echoes have the same weight Posse et al. (1999); Poser et al. (2006); Gowland and Bowtell (2007). Poser et al. (2006) found that matched filter weighting provided slightly better dCNR than flat weighting, with both schemes performing slightly worse than a variation referred to as temporal BOLD sensitivity weighting. Using a different protocol, Kettinger et al. (2016) found that average dCNR values obtained with matched filter weighting were slightly higher than those obtained with either flat or temporal BOLD sensitivity weighting approaches, but concluded that all the weighting schemes provided similar group-level statistical performance. Recently, Heunis et al. (2021) found that various weighting schemes, including the matched filter weighting, provided comparable tSNR measures across the whole brain and selected regions of interest.

In this paper, we present a geometric perspective that provides further insight into the dependence of tSNR and dCNR on the weighting scheme. After reviewing the definitions of tSNR and dCNR, we examine the optimal solution for each metric and consider variants of the optimal solution, relating these variants to previously used weighting schemes. We then describe three major regimes of interest and show how insight into weight dependence in each regime can be gained by considering the geometric relation between the numerator and denominator terms of the metrics. Representative behavior of the metrics in each regime is demonstrated using example data from a multi-echo acquisition with three echoes, facilitating a 3D graphical view of the weight dependence. Preliminary versions of this work were presented in Liu et al. (2020ab).

\section{Theory}

\subsection{Review of definitions}

In this section we briefly review the definitions of CNR, tSNR, and dCNR (Poser et al., 2006. Triantafyllou et al. 2005). To simplify the presentation, the spatial dependence of all quantities is assumed but not explicitly stated in the notation. For a given time series $S$, the contrast-to-noise-ratio is defined as $\mathrm{CNR}=\frac{\Delta S}{\sigma}$ where $\Delta S$ is the signal change and $\sigma$ is the standard deviation. For a single-echo 
acquisition this can be rewritten as

$$
\mathrm{CNR}=\frac{\Delta S}{\bar{S}} \frac{\bar{S}}{\sigma}=\frac{\Delta S}{\bar{S}} \cdot \mathrm{tSNR}
$$

55

a single-echo acquisition is the product of the fractional signal change (equivalent to the percent signal change divided by 100) and tSNR.

For a multi-echo acquisition, the signal of interest is the weighted sum $S=\sum_{k=1}^{N_{E}} w_{k} S_{k}$, where $w_{k}$ and $S_{k}$ denote the weight and signal of the $k$ th echo, respectively, and $N_{E}$ is the number of echoes. From the basic definition of tSNR, we can write the multi-echo version as

$$
\operatorname{tSNR}=\frac{\operatorname{mean}\left(\sum_{k=1}^{N_{E}} w_{k} S_{k}\right)}{\operatorname{std}\left(\sum_{k=1}^{N_{E}} w_{k} S_{k}\right)}=\frac{\sum_{k=1}^{N_{E}} w_{k} \bar{S}_{k}}{\operatorname{std}\left(\sum_{k=1}^{N_{E}} w_{k} S_{k}\right)}
$$

where $\bar{S}_{k}$ is the temporal mean of the $k$ th echo.

To derive an expression for multi-echo CNR, we express the signal change term as $\Delta S=\sum_{k=1}^{N_{E}} w_{k} \Delta S_{k}$ where $\Delta S_{k}$ is the signal change of the $k$ th echo. As the fractional BOLD signal change for the $k$ th echo can be well approximated as the product $\Delta S_{k} / \bar{S}_{k} \approx-\Delta R_{2}^{*}$. TE $E_{k}$ of the change in effective transverse relaxation rate $-\Delta R_{2}^{*}$ and echo time $\mathrm{TE}_{k}$, the signal change term may be written as $\Delta S=-\Delta R_{2}^{*} \sum_{k=1}^{N_{E}} w_{k} \mathrm{TE}_{k} \bar{S}_{k}$ (Poser et al. 2006, Kundu et al. 2013). Dividing this term by the standard deviation of the signal yields

$$
\mathrm{CNR}=-\Delta R_{2}^{*} \cdot \frac{\sum_{k=1}^{N_{E}} w_{k} \mathrm{TE}_{k} \bar{S}_{k}}{\operatorname{std}\left(\sum_{k=1}^{N_{E}} w_{k} S_{k}\right)}=-\Delta R_{2}^{*} \cdot \mathrm{dCNR}
$$

where differential CNR is defined as

$$
\mathrm{dCNR}=\frac{\sum_{k=1}^{N_{E}} w_{k} \mathrm{TE}_{k} \bar{S}_{k}}{\operatorname{std}\left(\sum_{k=1}^{N_{E}} w_{k} S_{k}\right)} .
$$

In comparing Equations 2 and 4 , we can see that the expressions for tSNR and dCNR differ only in the addition of the echo time $\mathrm{TE}_{k}$ terms in the numerator for dCNR. If all the echo times are the same $\left(\mathrm{TE}_{k}=\mathrm{TE}\right)$, then $\mathrm{dCNR}=\mathrm{TE} \cdot \mathrm{tSNR}$. However, for an arbitrary choice of echo times, tSNR is not necessarily proportional to either dCNR or CNR for a multi-echo acquisition.

To facilitate geometric understanding, we rewrite tSNR and dCNR using matrix notation. We define w as the $N_{E} \times 1$ vector of weights and $\mathbf{S}$ as a $N_{E} \times N_{T}$ matrix where the $i$ th row contains the time series 
data from the $i$ th echo, and $N_{T}$ denotes the number of time points. With this notation $\mathbf{w}^{T} \mathbf{S}$ represents the weighted sum of the signals and

$$
\operatorname{tSNR}=\frac{\operatorname{mean}\left(\mathbf{w}^{T} \mathbf{S}\right)}{\operatorname{std}\left(\mathbf{w}^{T} \mathbf{S}\right)}=\frac{\mathbf{w}^{T} \overline{\mathbf{s}}}{\operatorname{std}\left(\mathbf{w}^{T} \mathbf{S}\right)}
$$

where $\overline{\mathbf{s}}$ is the $N_{E} \times 1$ vector consisting of the temporal means $\bar{S}_{k}$. In a similar fashion, we can write

$$
\mathrm{dCNR}=\frac{\operatorname{mean}\left(\mathbf{w}^{T} \mathbf{D}_{\tau} \mathbf{S}\right)}{\operatorname{std}\left(\mathbf{w}^{T} \mathbf{S}\right)}=\frac{\mathbf{w}^{T} \mathbf{D}_{\tau} \overline{\mathbf{s}}}{\operatorname{std}\left(\mathbf{w}^{T} \mathbf{S}\right)}
$$

where $\mathbf{D}_{\tau}=\operatorname{diag}\left(\mathrm{TE}_{1}, \mathrm{TE}_{2}, \cdots, \mathrm{TE}_{N_{E}}\right)$ is the diagonal matrix comprised of the echo times.

\subsection{Optimal Solutions}

To maximize tSNR, we can maximize its square

$$
(\mathrm{tSNR})^{2}=\frac{\mathbf{w}^{T} \overline{\mathbf{s}}^{T} \mathbf{w}}{\operatorname{var}\left(\mathbf{w}^{T} \mathbf{S}\right)}=\frac{\mathbf{w}^{T} \mathbf{A} \mathbf{w}}{\mathbf{w}^{T} \mathbf{\Sigma} \mathbf{w}}
$$

where $\mathbf{A}=\overline{\mathbf{s}} \overline{\mathbf{s}}^{T}$ and $\boldsymbol{\Sigma}$ denotes the covariance matrix of $\mathbf{S}$. The expression for (tSNR) ${ }^{2}$ has the form of a generalized Rayleigh quotient (GRQ) and attains a maximum value of $\overline{\mathbf{s}}^{T} \boldsymbol{\Sigma}^{-1} \overline{\mathbf{s}}$ when the weight vector has the form of an optimal matched filter $\mathbf{w}=\kappa \boldsymbol{\Sigma}^{-1} \overline{\mathbf{s}}$, where $\kappa$ is an arbitrary scalar Brennan and Reed, 1973: Monzingo et al. 2011, Jarrett et al., 2017). From the form of the GRQ, it is straightforward to show that $\frac{\partial}{\partial \mathbf{w}}(\mathrm{tSNR})^{2}=0$ when $\mathbf{A}$ is a scalar multiple of $\boldsymbol{\Sigma}$. Thus, we expect that tSNR will be relatively insensitive to the form of $\mathbf{w}$ when $\mathbf{A} \sim \boldsymbol{\Sigma}$.

For dCNR, the corresponding GRQ is

$$
(\mathrm{dCNR})^{2}=\frac{\mathbf{w}^{T} \mathbf{D}_{\tau} \overline{\mathbf{s}}^{T} \mathbf{D}_{\tau} \mathbf{w}}{\operatorname{var}\left(\mathbf{w}^{T} \mathbf{S}\right)}=\frac{\mathbf{w}^{T} \mathbf{B} \mathbf{w}}{\mathbf{w}^{T} \mathbf{\Sigma} \mathbf{w}}
$$

where $\mathbf{B}=\mathbf{D}_{\tau} \overline{\mathbf{s}} \overline{\mathbf{s}}^{T} \mathbf{D}_{\tau}$. The GRQ attains its maximal value $\overline{\mathbf{s}}^{T} \mathbf{D}_{\tau} \boldsymbol{\Sigma}^{-1} \mathbf{D}_{\tau} \overline{\mathbf{s}}$ when $\mathbf{w}=\kappa \boldsymbol{\Sigma}^{-1} \mathbf{D}_{\tau} \overline{\mathbf{s}}$. Furthermore, the GRQ and hence dCNR are both fairly insensitive to the choice of weights when $\mathbf{B} \sim \boldsymbol{\Sigma}$. Since both tSNR and dCNR are invariant with respect to the value of $\kappa$, we will drop this scalar term for the remainder of the paper.

Under certain conditions, previously proposed weighting schemes can achieve optimal tSNR or dCNR.

For example, with the assumption that the random noise components have equal variance and are uncorrelated across the echoes (i.e. $\Sigma=\sigma^{2} \mathbf{I}$ ), the optimal weight vector for dCNR has the form $\mathbf{w}=\mathbf{D}_{\tau} \overline{\mathbf{s}}$ (i.e. $w_{k}=\mathrm{TE}_{k} \bar{S}_{k}$ ), which is the BOLD Sensitivity weight solution examined in Kettinger et al. (2016). This is equivalent to the matched filter solution proposed in Posse et al. (1999) when the signal means 
exhibit $T_{2}^{*}$ weighting (i.e. $\left.\bar{S}_{k} \propto \exp \left(-\mathrm{TE}_{k} / T_{2}^{*}\right)\right)$, as would be the case for most ME-fMRI acquisitions. For tSNR maximization, the assumption $\Sigma=\sigma^{2} \mathbf{I}$ leads to an optimal weight vector of the form $\mathbf{w}=\overline{\mathbf{s}}$ (i.e. $w_{k}=\bar{S}_{k}$ ), which is analogous to the exponential weighting considered by Posse et al. (1999).

If the noise is uncorrelated with unequal variances across echoes (i.e. $\boldsymbol{\Sigma}=\boldsymbol{\Lambda}=\operatorname{diag}\left(\sigma_{1}^{2}, \sigma_{2}^{2}, \cdots, \sigma_{N E}^{2}\right)$ ), then the optimal dCNR weight vector has the form $\mathbf{w}=\boldsymbol{\Lambda}^{-1} \mathbf{D}_{\tau} \overline{\mathbf{s}}$ (i.e. $w_{k}=\mathrm{TE}_{k} \bar{S}_{k} / \sigma_{k}^{2}$ ). This solution is similar to but not equivalent to the temporal BOLD sensitivity weighting $\mathbf{w}=\boldsymbol{\Lambda}^{-1 / 2} \mathbf{D}_{\tau} \overline{\mathbf{s}}$ (i.e. $\left.w_{k}=\mathrm{TE}_{k} \bar{S}_{k} / \sigma_{k}\right)$ proposed in Poser et al. (2006) and further examined in Kettinger et al. (2016). For tSNR maximization, the optimal weight vector when $\boldsymbol{\Sigma}=\boldsymbol{\Lambda}$ is $\mathbf{w}=\boldsymbol{\Lambda}^{-1} \overline{\mathbf{s}}$ (i.e. $w_{k}=\bar{S}_{k} / \sigma_{k}^{2}$ ), which is similar to but not equivalent to the tSNR weighting $\mathbf{w}=\boldsymbol{\Lambda}^{-1 / 2} \overline{\mathbf{s}}$ (i.e. $w_{k}=\bar{S}_{k} / \sigma_{k}$ ) studied in Kettinger et al. (2016).

The various options described above for the weight vector are summarized in Table 1 . The first four options consist of the tSNR optimal solution followed by three tSNR weight variants listed in order of decreasing similarity to the optimal solution. The last five options consist of the dCNR optimal solution followed by four dCNR weight variants listed in order of decreasing similarity to the optimal solution. In addition, we also consider a flat weighting of the echoes that has been examined in prior studies (Posse et al., 1999; Kettinger et al., 2016; Gowland and Bowtell, 2007).

\subsection{Geometric View}

In this section we consider three major regimes of interest with regards to the weight dependence of the sensitivity metrics.

1. tSNR Robust Regime: tSNR is relatively robust to the choice of weights with most variants achieving close to optimal performance, while dCNR shows a substantial dependence on the weights with most variants achieving significantly lower than optimal performance.

2. dCNR Robust Regime: dCNR is relatively robust to the choice of weights with most variants achieving close to optimal performance, while tSNR exhibits a substantial dependence on the weights with most variants achieving significantly lower than optimal performance.

3. Within-type Robust Regime: In this regime, both tSNR and dCNR are nearly optimal for weight variants that are similar to their respective optimal solution and exhibit moderately high performance for weight variants that are similar to the optimal solution for the other metric.

The tSNR and dCNR robust regimes are characterized by a high degree of similarity between their respective numerator matrices and the covariance matrix $\boldsymbol{\Sigma}$, with $\mathbf{A} \sim \boldsymbol{\Sigma}$ for the tSNR robust regime and 
$\mathbf{B} \sim \boldsymbol{\Sigma}$ for the dCNR robust regime. Since $\mathbf{A}$ and $\mathbf{B}$ are both symmetric rank-one matrices formed as the outer products of a vector ( $\overline{\mathbf{s}}$ and $\mathbf{D}_{\tau} \overline{\mathbf{s}}$, respectively) with its transpose, a high degree of similarity can be achieved when $\boldsymbol{\Sigma}$ is well-approximated by a rank-one matrix. This occurs when there is a dominant eigenvalue $\lambda_{1} \gg \lambda_{i \neq 1}$ such that the covariance matrix may be approximated as $\boldsymbol{\Sigma} \approx \lambda_{1} \mathbf{v}_{1} \mathbf{v}_{1}^{T}$, where $\mathbf{v}_{1}$ is the corresponding eigenvector.

To proceed, it is useful to examine the geometry of the numerator and denominator terms of the metrics in Equations 5 and 6 . The numerator term is the inner product of the weight vector $\mathbf{w}$ with either the mean signal vector $\overline{\mathbf{s}}$ for tSNR or the TE-weighted mean signal vector $\mathbf{D}_{\tau} \overline{\mathbf{s}}$ for dCNR. If the numerator term is normalized to have a maximum of 1.0, then the inner product as a function of weight can be written as either $\left|\cos \left(\theta_{t S N R}(\mathbf{w})\right)\right|$ or $\left|\cos \left(\theta_{d C N R}(\mathbf{w})\right)\right|$, where $\theta_{t S N R}(\mathbf{w})$ and $\theta_{d C N R}(\mathbf{w})$ are the polar angles of $\mathbf{w}$ referenced to the vectors $\overline{\mathbf{s}}$ and $\mathbf{D}_{\tau} \overline{\mathbf{s}}$ for tSNR and dCNR, respectively. Examples showing this cosine dependence are shown in Figure 1 panels (a) and (d) for a representative voxel, where the color of each point on the sphere indicates the value of the normalized numerator term given a unit-norm weight vector pointing in that direction. When the covariance matrix has a dominant eigenvalue, a similar argument can be used to show that the denominator term can be well approximated as $\left|\cos \left(\theta_{\mathbf{v}_{1}}(\mathbf{w})\right)\right|$ where $\theta_{\mathbf{v}_{1}}(\mathbf{w})$ is the polar angle of $\mathbf{w}$ referenced to $\mathbf{v}_{1}$. Figure $1 \mathrm{~b}$ shows an example of the corresponding surface plot for the representative voxel in which the dominant eigenvalue is 18.8 times larger than the second highest eigenvalue.

The weight dependence of each metric reflects the ratio of the numerator and denominator surface plots. When the angle $\Delta \theta$ between the dominant eigenvector $\mathbf{v}_{1}$ and the numerator reference vector (either $\overline{\mathbf{s}}$ or $\mathbf{D} \overline{\mathbf{s}}$ ) is relatively small, then the numerator and denominator surface plots will exhibit similar cosine dependencies on the weights and the corresponding metric is relatively insensitive to $\mathbf{w}$. As an example, for the surface plots in Figure 1 panels (b) and (a) there is a relatively small angle $\Delta \theta=3.5^{\circ}$ between the reference vectors $\mathbf{v}_{1}$ and $\mathbf{D}_{\tau} \overline{\mathbf{s}}$, yielding a dCNR surface plot (panel c) that is relatively insensitive to the weights. However, since the angle between $\mathbf{v}_{1}$ and $\mathbf{D}_{\tau} \overline{\mathbf{s}}$ is small, this means that the angle between $\mathbf{v}_{1}$ and $\overline{\mathbf{s}}$ will be relatively large when the echo times span a range of values, as is typical for most multi-echo acquisitions. For the surface plots in panels (b) and (d), the angle between $\mathbf{v}_{1}$ and $\overline{\mathbf{s}}$ is $\Delta \theta=32.6^{\circ}$. The numerator and denominator terms exhibit cosine dependencies with significantly different directions, yielding a tSNR surface plot (panel e) that is highly sensitive to the weights. Thus, this example illustrates a key trade-off observed in the dCNR robust regime. When one metric (e.g. $\mathrm{dCNR}$ ) is robust to the weights, the other metric (tSNR) will exhibit a high degree of dependence on the 
weights. The opposite tradeoff is observed in the tSNR robust regime, as described further in the Results section.

In the within-type robustness regime, the covariance matrix does not have a dominant eigenvalue and the denominator term shows a weak dependence on the weights. Figure $1 \mathrm{f}$ shows an example in which there is a fairly uniform spread of eigenvalues (normalized range of 1.00 to 1.25) leading to a relatively uniform denominator surface plot without a strong preferred direction. For this case, the tSNR metric shown in panel $(\mathrm{h})$ primarily reflects the cosine dependence of the tSNR numerator term shown in panel (f). Furthermore, in this regime the covariance matrix can be roughly approximated $\boldsymbol{\Sigma} \sim \mathbf{I}$ by the identity matrix. With this approximation, the tSNR weight variants (tdiag, tsnr, swt) approach the tSNR optimal solution $\mathbf{w} \sim \overline{\mathbf{s}}$ while the dCNR weight variants (ddg, tBS, BS, t2wt) approach the dCNR optimal solution $\mathbf{w} \sim \mathbf{D}_{\tau} \overline{\mathbf{s}}$. Thus, within each type there is a high degree of robustness to the weight selection when using a weight variant of that type (i.e. nearly optimal dCNR values obtained when using dCNR weight variants). Finally, because the metrics exhibit a cosine dependence on the weights, the performance when using a weight variant of the other type (e.g. decrease in dCNR when using a tSNR weight variant) can be approximated as the cosine of the angle between $\overline{\mathbf{s}}$ and $\mathbf{D}_{\tau} \overline{\mathbf{s}}$. These concepts will be demonstrated in greater detail in the Results section.

\subsection{Equivalence of normalized quantities}

A specific case of the trade-off observed in the tSNR and dCNR robust regimes occurs when comparing tSNR computed using the dCNR optimal weight versus dCNR computed using the tSNR optimal weight. When these metrics are each normalized by their respective maximum values (obtained by using the tSNR and dCNR optimal weights, respectively), it can be shown that

$$
\frac{\left.\mathrm{tSNR}\right|_{\mathbf{w}=\boldsymbol{\Sigma}^{-1} \mathbf{D}_{\tau} \overline{\mathbf{s}}}}{\left.\mathrm{tSNR}\right|_{\mathbf{w}=\boldsymbol{\Sigma}^{-1} \overline{\mathbf{s}}}}=\frac{\left.\mathrm{dCNR}\right|_{\mathbf{w}=\boldsymbol{\Sigma}^{-1} \overline{\mathbf{s}}}}{\left.\mathrm{dCNR}\right|_{\mathbf{w}=\boldsymbol{\Sigma}^{-1} \mathbf{D}_{\tau} \overline{\mathbf{s}}}}=\frac{\overline{\mathbf{s}}^{T} \boldsymbol{\Sigma}^{-1} \mathbf{D}_{\tau} \overline{\mathbf{s}}}{\sqrt{\overline{\mathbf{s}}^{T} \boldsymbol{\Sigma}^{-1} \overline{\mathbf{s}}} \sqrt{\overline{\mathbf{s}}^{T} \mathbf{D}_{\tau} \boldsymbol{\Sigma}^{-1} \mathbf{D}_{\tau} \overline{\mathbf{s}}}} \leq 1
$$

where the inequality follows from the Cauchy-Schwarz inequality $\left(\left|\mathbf{u}^{T} \mathbf{v}\right|^{2} \leq\left(\mathbf{u}^{T} \mathbf{u}\right)\left(\mathbf{v}^{T} \mathbf{v}\right)\right)$. In other words, the normalized tSNR obtained with the dCNR optimal weight is equal to the normalized dCNR obtained with the tSNR optimal weight, and both quantities are less than or equal to 1.

\section{Methods}

To provide representative examples of the geometric view, resting-state fMRI data (eyes-closed) were acquired under Institutional Review Board approval. One healthy male was scanned on a Discovery 
MR750 3T system (GE Healthcare) with a 32-channel receive head coil (Nova Medical) and a multi-echo

\section{1. $t S N R$ robust regime}

As an example of behavior in the tSNR robust regime, Figure 3 a shows tSNR and dCNR values as a function of weight variant for representative voxel C. For all panels in the figure, the tSNR and dCNR values are normalized by their corresponding maximum values of $\sqrt{\overline{\mathbf{s}}^{T} \boldsymbol{\Sigma}^{-1} \overline{\mathbf{s}}}$ and $\sqrt{\overline{\mathbf{s}}^{T} \mathbf{D}_{\tau} \boldsymbol{\Sigma}^{-1} \mathbf{D}_{\tau} \overline{\mathbf{s}}}$, which robust to the choice of weight vector (ranging from 0.97 to 1.0), with the exception of the noticeably lower value of 0.70 for the dCNR optimal weight. In contrast, the dCNR values exhibit a greater degree of 
dependence on the choice of weight with values ranging from 0.53 to 1.0. The dotted black line highlights the equivalence stated previously in Equation 9 i.e. the normalized tSNR when using the optimal dCNR weight is equal to the normalized dCNR when using the optimal tSNR weight. (This equivalence is also highlighted for the other regimes shown in rows b and c).

A geometric view of the tSNR robust regime is provided in the left column of Figure 4 . In this figure the first and last rows show the absolute values of the normalized tSNR and dCNR values, respectively, plotted on the surface of a sphere, where each point of the sphere corresponds to a weight vector with unit norm. The tSNR and dCNR weight variants are indicated with brown squares and magenta circles, respectively, and the flat weight vectors are indicated by green diamonds. The second and fourth rows show the absolute values of the tSNR $\left(\sqrt{\mathbf{w}^{T} \mathbf{A w}}\right)$ and $\mathrm{dCNR}\left(\sqrt{\mathbf{w}^{T} \mathbf{B w}}\right)$ numerator terms, respectively, while the third row show the common denominator term $\sqrt{\mathbf{w}^{T} \mathbf{\Sigma} \mathbf{w}}$. As described in detail in the figure caption, the symbols on the surface indicate the positions of the different weight variants.

Focusing on the left column for voxel $\mathrm{C}$, both the tSNR and dCNR numerator surfaces (panels $\mathrm{d}$ and $\mathrm{j}$ ) exhibit the cosine dependence described in Section 2.3 . In addition, the denominator surface (panel g) also exhibits a cosine dependence because the covariance matrix has a dominant eigenvalue that is 7.1 times larger than the second highest eigenvalue. As the angle between the associated dominant eigenvector and the vector $\overline{\mathbf{s}}$ is relatively small $\left(\Delta \theta=6.3^{\circ}\right)$, the tSNR numerator and denominator surfaces show a similar weight dependence, and the ratio of the two surfaces leads to a tSNR surface plot (panel a) that is relatively insensitive to the weights, consistent with the behavior shown by the green line in Figure $3 \mathrm{a}$. In contrast, the principal directions of the dCNR numerator and denominator are not well aligned $\left(\Delta \theta=33.4^{\circ}\right)$, so that the ratio of these two terms results in a dCNR surface plot (panel $\mathrm{m}$ ) that is sensitive to $\mathbf{w}$, consistent with the behavior shown by the red line in Figure 3 a.

\section{2. $d C N R$ robust regime}

To demonstrate behavior in the dCNR robust regime, Figure $3 \mathrm{~b}$ shows tSNR and dCNR values as a function of weight variant for representative voxel $\mathrm{G}$. The dCNR values are relatively robust to the choice of weight variant (ranging from 0.96 to 1.0), with the exception of the noticeably lower value of 0.54 for the tSNR optimal weight (which is equal to the tSNR value obtained with the dCNR optimal weight, as indicated by the black dotted line). In contrast, the tSNR values exhibit a high degree of weight sensitivity with values ranging from 0.34 to 1.0 . 
The geometric view of this regime was previously described in Section 2.3 and Figure 1 . For completeness, it is presented in a slightly different format in the middle column of Figure 44. The dCNR numerator (panel k) and denominator (panel h) exhibit similar cosine dependencies, reflecting the relatively small angle $\left(\Delta \theta=3.5^{\circ}\right)$ between $\mathbf{D}_{\tau} \overline{\mathbf{s}}$ and the dominant eigenvector of the covariance matrix. As a result, the ratio results in a dCNR surface plot (panel $\mathrm{n}$ ) that is relatively insensitive to $\mathbf{w}$. In contrast, the principal directions of the tSNR numerator (panel e) and denominator surface plots are not well aligned $\left(\Delta \theta=32.6^{\circ}\right)$, so that the ratio of results in the tSNR surface plot (panel b) that is highly dependent on $\mathbf{w}$.

\subsection{Within-type robust regime}

As an example of behavior in the within-type robust regime, Figure 3 ; shows tSNR and dCNR values across weight variants for voxel $\mathrm{W}$. The normalized tSNR values for the tSNR optimal weight variants (topt, tdiag, tsnr, swt) are within 0.005 of the maximum value of 1.000 , reflecting the fact that all of the variants approach the optimal solution $\mathbf{w} \sim \overline{\mathbf{s}}$ when $\boldsymbol{\Sigma} \sim \mathbf{I}$. Similarly, the dCNR values for the dCNR optimal weight variants (dopt, ddg, tBS, BS, t2wt) are within 0.003 of the maximum value of 1.000 , reflecting the fact that all of these variants approach the optimal solution $\mathbf{w} \sim \mathbf{D}_{\tau} \overline{\mathbf{s}}$. In considering the behavior of the other points, we note that both the tSNR values using the dCNR weight variants (ddg, tBS, BS, and t2wt) and the dCNR values using the tSNR weight variants (tdg, tnsr, and swt) lie fairly close to the dotted black equivalent value line (corresponding to tSNR with optimal dCNR weight (dopt) and dCNR with optimal tSNR weight (topt)).

The geometric view of tSNR in this regime was previously described in Section 2.3 and Figure 1 In the right column of Figure 4 we expand upon the prior description. Due to the fairly uniform spread of the covariance matrix eigenvalues (normalized range of 1.0 to 1.3), the denominator surface (panel i) is fairly uniform. As a result, both the tSNR (panel c) and dCNR (panel o) surface plots reflect the cosine dependence of their respective numerator terms (panels $\mathrm{f}$ and $\mathrm{l}$ ). In contrast to the spread in weight variant positions observed in the other regimes, the weight variants for voxel $\mathrm{W}$ are fairly tightly clustered within each variant type. As noted above, the tSNR and dCNR weight variants approach their respective optimal solutions (indicated with asterisks) in this regime, so that for tSNR in panel (c) the tSNR weight variants appear in the brown cluster, while for dCNR in panel (o) the dCNR weight variants appear in the magenta cluster. Each of these surfaces also contains a secondary cluster, corresponding to tSNR values when using dCNR weight variants (magenta cluster in panel c) and dCNR values when 
using tSNR weight variants (brown cluster in panel o). These are offset from the optimal clusters by an angle that is roughly the difference $\Delta \theta_{W}=\theta_{\overline{\mathbf{s}}}-\theta_{\mathbf{D}_{\tau} \overline{\mathbf{s}}}$ between the angles of $\overline{\mathbf{s}}$ and $\mathbf{D}_{\tau} \overline{\mathbf{s}}$. The cosine of this difference angle $\cos \left(\Delta \theta_{W}\right)=0.87$ indicates reflects the reduced performance of the secondary cluster as compared to the maximum value of 1.0 obtained within the optimal cluster. In addition, it approximates the equivalent value shown by the black dotted line in Figure 3 ; , because the expression in Equation 9 is well-approximated by $\cos \left(\Delta \theta_{W}\right)$ when $\boldsymbol{\Sigma} \sim \mathbf{I}$.

\section{Discussion}

We have presented a geometric view to characterize the weight dependence of tSNR and dCNR in multi-echo fMRI. Using this view, we have described three major regimes of behavior: a tSNR robust regime, a dCNR robust regime, and a within-type robust regime. The tSNR and dCNR robust regimes are characterized by covariance matrices with a dominant eigenvector. In the tSNR robust regime there is a high degree of similarity between the dominant eigenvector and the vector $\overline{\mathbf{s}}$ of signal means, whereas in the dCNR robust regime there is a high degree of similarity between the dominant eigenvector and the vector $\mathbf{D}_{\tau} \overline{\mathbf{s}}$ of TE-weighted signal means. Since $\overline{\mathbf{s}}$ and $\mathbf{D}_{\tau} \overline{\mathbf{s}}$ are generally not well-aligned, there is a fundamental trade-off observed within both the tSNR and dCNR robust regimes. When one of the metrics (e.g. dCNR) is relatively robust to the choice of the weight vector, the other metric (e.g. tSNR) will tend to exhibit a strong dependence on the weights. In the within-type regime, the covariance matrix does not have a dominant eigenvector and is similar in structure to the identity matrix. Within this regime, both metrics exhibit nearly optimal performance when using variants of their respective optimal weight vector. In addition, the reduced performance when using other variants is well approximated by the cosine of the angle between $\overline{\mathbf{s}}$ and $\mathbf{D}_{\tau} \overline{\mathbf{s}}$. Finally, we note that for the within-type regime both the matched filter solution proposed in Posse et al. (1999) and the BOLD Sensitivity weight solution examined in Kettinger et al. (2016) approach the dCNR optimal solution, and the exponential weighting considered by Posse et al. (1999) approaches the tSNR optimal solution.

In the tSNR robust regime, the similarity between $\overline{\mathbf{s}}$ and the dominant eigenvector of the covariance matrix indicates that the noise in each echo scales with the mean signal and may be considered roughly "signal-like" (Gowland and Bowtell, 2007). In addition, since the covariance matrix can be wellapproximated as a rank one matrix, the noise correlation between different echoes will be proportional to the product of the signal means of the echoes. For the dCNR robust regime, the similarity between $\mathbf{D}_{\tau} \overline{\mathbf{s}}$ 
and the dominant eigenvector of the covariance matrix indicates that the noise in each echo scales with the expected BOLD signal change and may be considered roughly "BOLD-like" Gowland and Bowtell 2007). Furthermore, the noise correlation between different echoes will be proportional to the product of the expected BOLD signal changes of the echoes. Finally, in the within-type robust regime, the similarity between the covariance matrix and the identity matrix indicates that the noise is largely uncorrelated between echoes with a variance that is roughly independent of the echo time. These characteristics indicate that thermal noise is dominant in this regime (Posse et al., 1999, Poser et al., 2006; Gowland and Bowtell, 2007). Taken together, these observations suggest that tSNR will tend to be fairly robust to the choice of weights in brain regions where the noise is either "signal-like" or thermal-noise dominated, but less robust in regions where the noise is "BOLD-like." On the other hand, dCNR will be fairly robust in regions where the noise is either "BOLD-like" or thermal-noise dominated, and less robust in regions where the noise is "signal-like."

In this paper, we used representative voxels from a multi-echo fMRI acquisition to illustrate the geometric view and the major regimes of behavior. The tSNR robust, dCNR robust, and within-type robust regimes were represented using voxels from cerebrospinal fluid, gray matter, and white matter, respectively. It is important to note that we are not making a general claim that voxels from these tissue types will always represent the different regimes. Instead, the correspondence between tissue type and operating regime will depend on the details of the acquisition used, such as spatial resolution, the choice of echo times, and magnetic field strength, as well as additional factors such as magnetic field inhomogeneities. For example, a decrease in voxel volume is expected to lead to a reduction in the ratio of physiological noise to thermal noise fluctuations (Triantafyllou et al., 2005, Bodurka et al., 2007) and could potentially nudge a gray matter voxel away from the dCNR robust regime towards the withintype regime. In regions with pronounced magnetic field inhomogeneities, signal decay due to intra-voxel dephasing may dominate over BOLD-like changes, potentially moving a gray matter voxel from the dCNR robust regime to the tSNR robust regime. Further work to characterize the operating regimes across a range of acquisition parameters and conditions would be of interest.

The insights provided by the geometric view can serve to better guide the selection of weights, taking into account the metrics and voxels that are of primary interest. For example, if a researcher is primarily interested in $\mathrm{dCNR}$ and determines that the voxels that are of interest (e.g. gray matter) largely belong to the dCNR robust regime, then most weight variants will be sufficient to achieve high dCNR. However, if tSNR is the primary metric of interest, then the researcher may want to consider using the tSNR 
optimal weight vector, since other choices are likely to lead to significantly lower tSNR. On the other hand, if the voxels of interest largely belong to the within-type robust regime, a researcher may want to choose the flat weight vector since it lies between the clusters of dCNR and tSNR weight variants and thus represents a reasonable compromise that can achieve relatively high performance for both metrics.

In this work we characterized the robustness of tSNR and dCNR to the choice of weights. It is possible that tSNR and dCNR may also reflect differences in robustness to other processing choices, such as the choice of approach for physiological noise reduction. Future work is needed to explore these potential differences. For the geometric view presented in this paper, we considered a multi-echo acquisition with 3 echoes, facilitating a 3D graphical view of the weight dependence. As the arguments put forth in the Theory section do not depend on the number of echoes $N_{E}$, the geometric view is applicable to other values of $N_{E}$ but is difficult to visualize in higher dimensions.

As mentioned in the Introduction, both tSNR and dCNR have been used in prior studies to characterize the sensitivity of multi-echo acquisition and analysis approaches. Drawing general conclusions from the prior work can be complicated by the fact that a given study typically only uses one of these metrics. Furthermore, it is not unusual for a study to use one metric (e.g. tSNR) with a weighting scheme optimized for another metric (e.g. optimal matched-filter weighting designed to optimize CNR) (Kundu et al. 2013, Cohen et al., 2017; Heunis et al., 2021). In light of the present findings, researchers may want to consider using the framework presented here to characterize the operating regimes observed in their data and to report both tSNR and dCNR metrics. Adopting such practices could greatly facilitate the comparison of results across studies.

\section{Data Availability Statement}

The data and analysis code for this paper will be made available at https://osf.io/7awdm/ upon publication.

\section{Acknowledgements}

We thank Conan Chen, Baolian Yang, and Robert Bussell for their assistance with this work. This work was supported in part by a research grant from GE Healthcare. 


\section{References}

\section{References}

Kundu, P., Brenowitz, N.D., Voon, V., Worbe, Y., Vértes, P.E., Inati, S.J., Saad, Z.S., Bandettini, P.A., Bullmore, E.T., 2013. Integrated strategy for improving functional connectivity mapping using multiecho fMRI. Proceedings of the National Academy of Sciences of the United States of America 110, 16187-16192. 
Kundu, P., Voon, V., Balchandani, P., Lombardo, M., Poserd, B., Bandettini, P., 2017. Multi-echo fMRI: A review of applications in fMRI denoising and analysis of BOLD signals. NeuroImage 154, 59-80.

Liu, T.T., Li, B., Chen, C., Fernandez, B., Yang, B., Banerjee, S., 2020a. Temporal SNR in multiecho fMRI and its dependence on the choice of weights, in: Proceedings of the 28th Annual Meeting of the ISMRM, Paris. p. 3820.

Liu, T.T., Li, B., Chen, C., Fernandez, B., Yang, B., Banerjee, S., 2020b. Weight Sensitivity of Temporal SNR Metrics in multi-echo fMRI. bioRxiv , 2020.04.20.049460doi:10.1101/2020.04.20.049460.

Monzingo, R., Haupt, R., Miller, T., 2011. Introduction to Adaptive Arrays. 2nd ed., Scitech Publishing, Raleigh, NC.

Parrish, T.B., Gitelman, D.R., LaBar, K.S., Mesulam, M.M., 2000. Impact of signal-to-noise on functional

MRI. Magnetic Resonance in Medicine 44, 925-932.

Poser, B.A., Versluis, M.J., Hoogduin, J.M., Norris, D.G., 2006. BOLD contrast sensitivity enhancement and artifact reduction with multiecho EPI: parallel-acquired inhomogeneity-desensitized fMRI. Magnetic resonance in medicine : official journal of the Society of Magnetic Resonance in Medicine / Society of Magnetic Resonance in Medicine 55, 1227-1235.

Posse, S., Wiese, S., Gembris, D., Mathiak, K., Kessler, C., Grosse-Ruyken, M.L., Elghahwagi, B., Richards, T., Dager, S.R., Kiselev, V.G., 1999. Enhancement of BOLD-contrast sensitivity by singleshot multi-echo functional MR imaging. Magnetic resonance in medicine : official journal of the Society of Magnetic Resonance in Medicine / Society of Magnetic Resonance in Medicine 42, 87-97.

Puckett, A.M., Bollmann, S., Poser, B.A., Palmer, J., Barth, M., Cunnington, R., 2018. Using multi-echo ganglia at ultra-high field (7T). NeuroImage 172, 886-895.

Triantafyllou, C., Hoge, R.D., Krueger, G., Wiggins, C.J., Potthast, A., Wiggins, G.C., Wald, L.L., 2005. Comparison of physiological noise at $1.5 \mathrm{~T}, 3 \mathrm{~T}$ and $7 \mathrm{~T}$ and optimization of fMRI acquisition parameters. NeuroImage 26, 243-250. 


\begin{tabular}{|c|c|c|c|}
\hline Method & Abbrev. & $\mathbf{w}$ & Comments \\
\hline tSNR Optimal & topt & $\boldsymbol{\Sigma}^{-1} \overline{\mathbf{s}}$ & \\
\hline tSNR Diagonal & $\operatorname{tdg}$ & $\boldsymbol{\Lambda}^{-1} \overline{\mathbf{s}}$ & tSNR optimal if $\boldsymbol{\Sigma}$ is diagonal \\
\hline tSNR Weighted & tsnr & $\Lambda^{-1 / 2} \overline{\mathbf{s}}$ & \\
\hline Signal Weighted & swt & $\overline{\mathbf{s}}$ & tSNR optimal if $\boldsymbol{\Sigma}=\sigma^{2} \mathbf{I}$ \\
\hline Flat & flat & 1 & \\
\hline dCNR Optimal & dopt & $\boldsymbol{\Sigma}^{-1} \mathbf{D}_{\tau} \overline{\mathbf{s}}$ & \\
\hline dCNR Diagonal & ddg & $\boldsymbol{\Lambda}^{-1} \mathbf{D}_{\tau} \overline{\mathbf{s}}$ & dCNR optimal if $\boldsymbol{\Sigma}$ is diagonal \\
\hline Temporal BOLD Sensitivity & tBS & $\boldsymbol{\Lambda}^{-1 / 2} \mathbf{D}_{\tau} \overline{\mathbf{s}}$ & \\
\hline BOLD Sensitivity & BS & $\mathbf{D}_{\tau} \overline{\mathbf{s}}$ & dCNR optimal if $\boldsymbol{\Sigma}=\sigma^{2} \mathbf{I}$ \\
\hline$T_{2}^{*}$ Weighted & t2wt & $\mathbf{D}_{\tau} \exp \left(-\operatorname{diag}\left(\mathbf{D}_{\tau}\right) / T_{2}^{*}\right)$ & $\begin{array}{l}\text { dCNR optimal if } \boldsymbol{\Sigma}=\sigma^{2} \mathbf{I} \text { and } \\
\overline{\mathbf{s}} \propto \exp \left(-\operatorname{diag}\left(\mathbf{D}_{\tau}\right) / T_{2}^{*}\right)\end{array}$ \\
\hline
\end{tabular}

Table 1: Description of weight vectors $\mathbf{w}$ where $\mathbf{s}$ denotes the vector of signal means, $\boldsymbol{\Sigma}$ denotes the noise covariance matrix, $\boldsymbol{\Lambda}$ is the diagonal matrix with noise variances along the diagonal, $\mathbf{D}_{\tau}$ is the diagonal matrix composed of echo times on the diagonal, $\mathbf{I}$ is the identity matrix, and $\mathbf{1}$ is a column vector of 1 's. The $T_{2}^{*}$ weighted solution (also referred to in the literature as matched filter or optimal combination) was originally proposed in Posse et al. (1999) and is equivalent to the BOLD Sensitivity weight examined in Kettinger et al. (2016) when the mean signal exhibits monoexponential $T_{2}^{*}$ decay. Temporal BOLD sensitivity weighting was proposed by Poser et al. (2006) and tSNR weighting was proposed by Kettinger et al. (2016). The tSNR diagonal and dCNR diagonal weight vector are variants of the tSNR and dCNR optimal weight vectors, respectively, obtained by ignoring the off-diagonal elements of $\boldsymbol{\Sigma}$. These are similar in form but not equivalent to the temporal BOLD sensitivity and tSNR weight vectors. The signal weighted solution is analogous to the exponential weighting considered by Posse et al. (1999). 

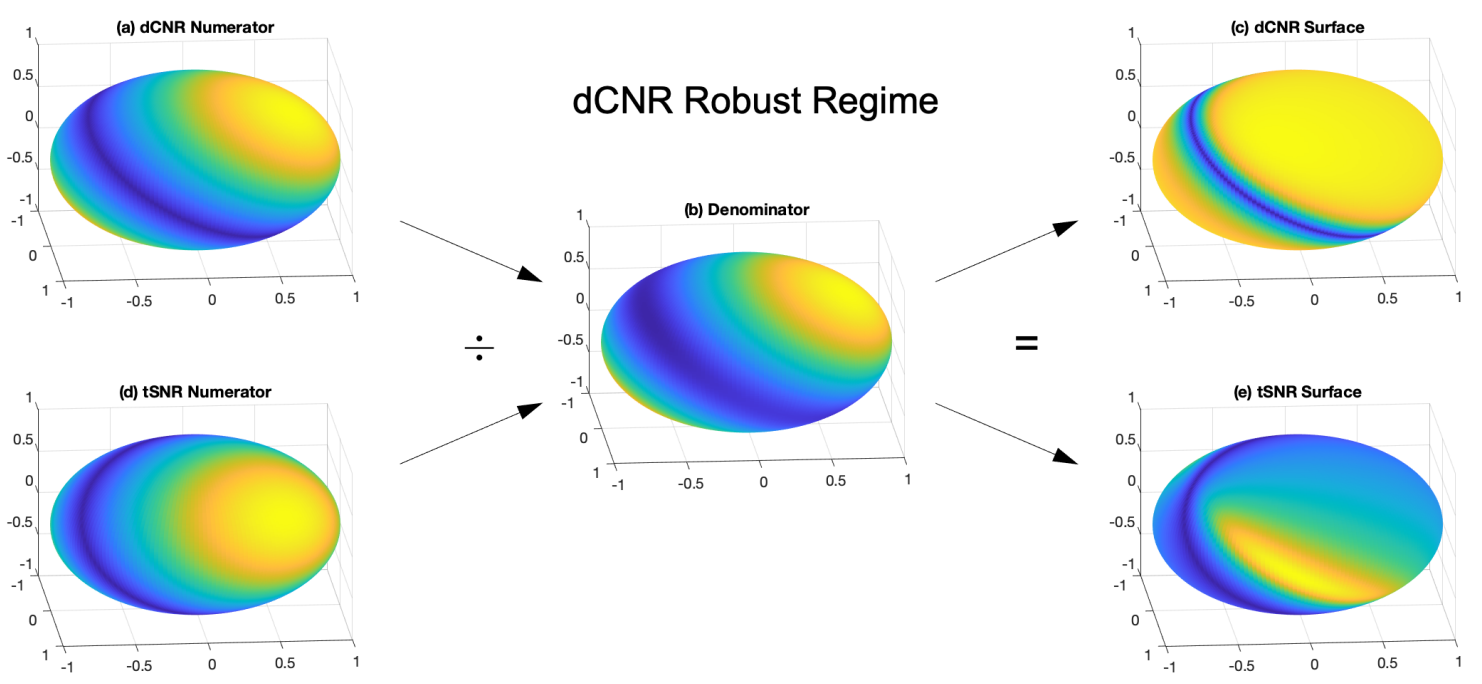

\section{Within-Type Robust Regime}
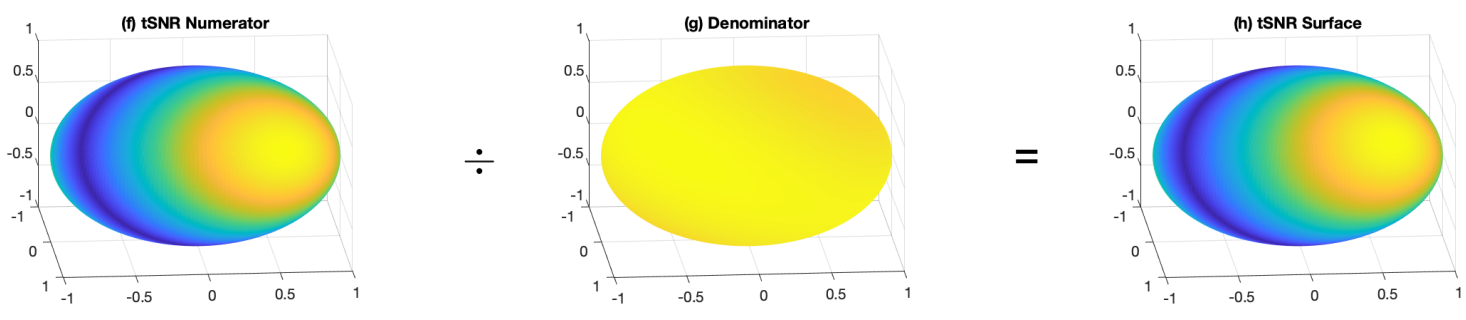

Figure 1: Geometric view of the dCNR robust regime and the within-type robust regime. The spherical surface plots indicate the value of the displayed quantities normalized by their maximum value, with each point on a sphere corresponding to a weight vector with unit norm. In the dCNR robust regime, the (a) dCNR numerator, the (d) tSNR numerator, and the (b) common denominator surface plots all exhibit a cosine dependence on the weights. The dCNR and tSNR surface plots are obtained by dividing the respective numerator terms by the common denominator terms. The robustness of the dCNR surface plot (panel c) reflects the relatively good alignment between the dCNR numerator and denominator surfaces. In contrast, the weight senstivity of the tSNR surface (panel e) reflects the misalignement between the tSNR numerator and denominator surfaces. In the within-type robust regime, the denominator surface (panel g) is fairly uniform, so that the tSNR surface (panel h) inherits the cosine dependence of the tSNR numerator surface (panel f). 
bioRxiv preprint doi: https://doi.org/10.1101/2022.02.23.481688; this version posted February 25, 2022. The copyright holder for this preprint (which was not certified by peer review) is the author/funder, who has granted bioRxiv a license to display the preprint in perpetuity. It is made available under aCC-BY-NC-ND 4.0 International license.

(a) Cosine Similarity between $\mathbf{A}=\overline{\mathbf{s}} \overline{\mathbf{s}}^{T}$ and $\boldsymbol{\Sigma}$

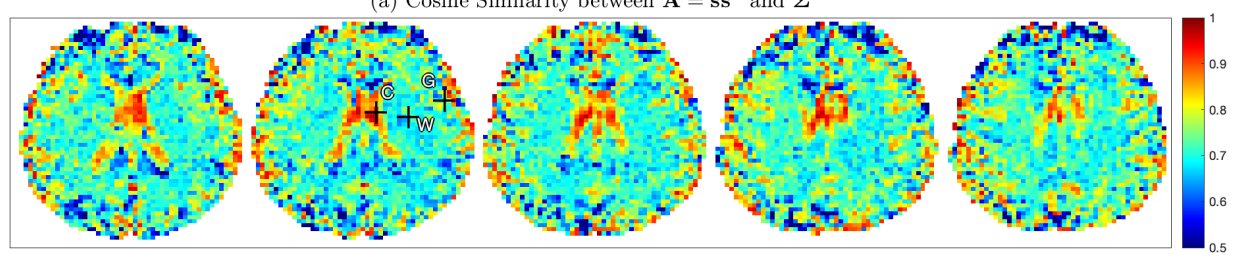

(b) Cosine Similarity between $\mathbf{B}=\mathbf{D}_{\tau} \overline{\mathbf{s}}^{T} \mathbf{D}_{\tau}^{T}$ and $\boldsymbol{\Sigma}$

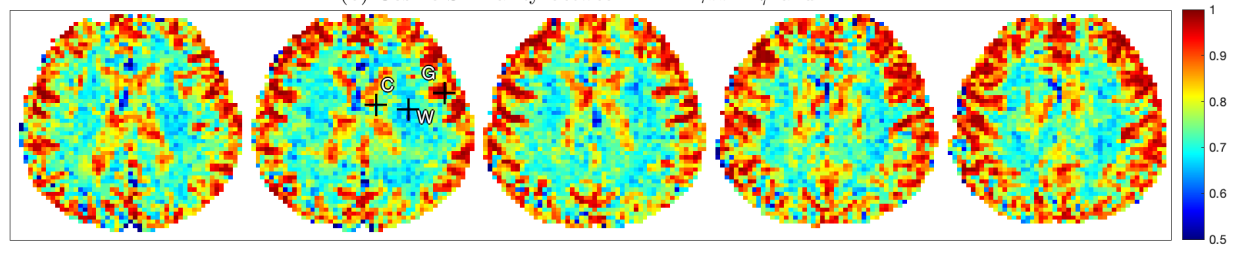

(c) Cosine Similarity between $\mathbf{I}$ and $\boldsymbol{\Sigma}$

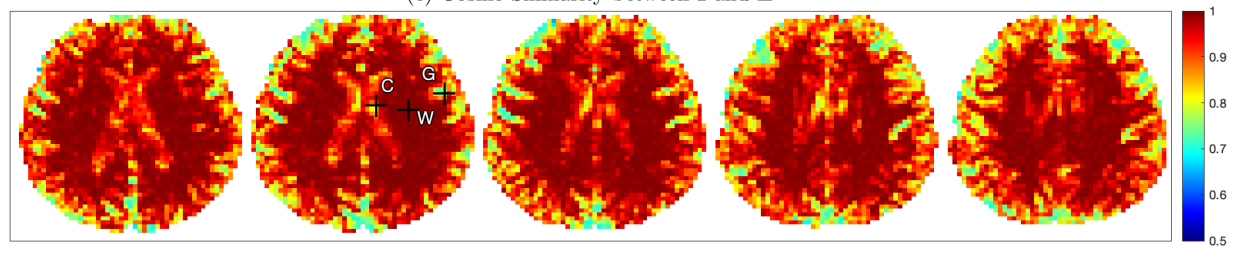

Figure 2: Maps of cosine similarity between the covariance matrix $\boldsymbol{\Sigma}$ and (a) tSNR numerator matrix $\mathbf{A}$, (b) dCNR numerator matrix B, and (c) the identity matrix I. Representative voxels for tSNR robust regime, dCNR robust regime, and within-type robust regime are marked with plus symbols and the letters C (CSF), G (gray matter), and W (white matter), respectively. 

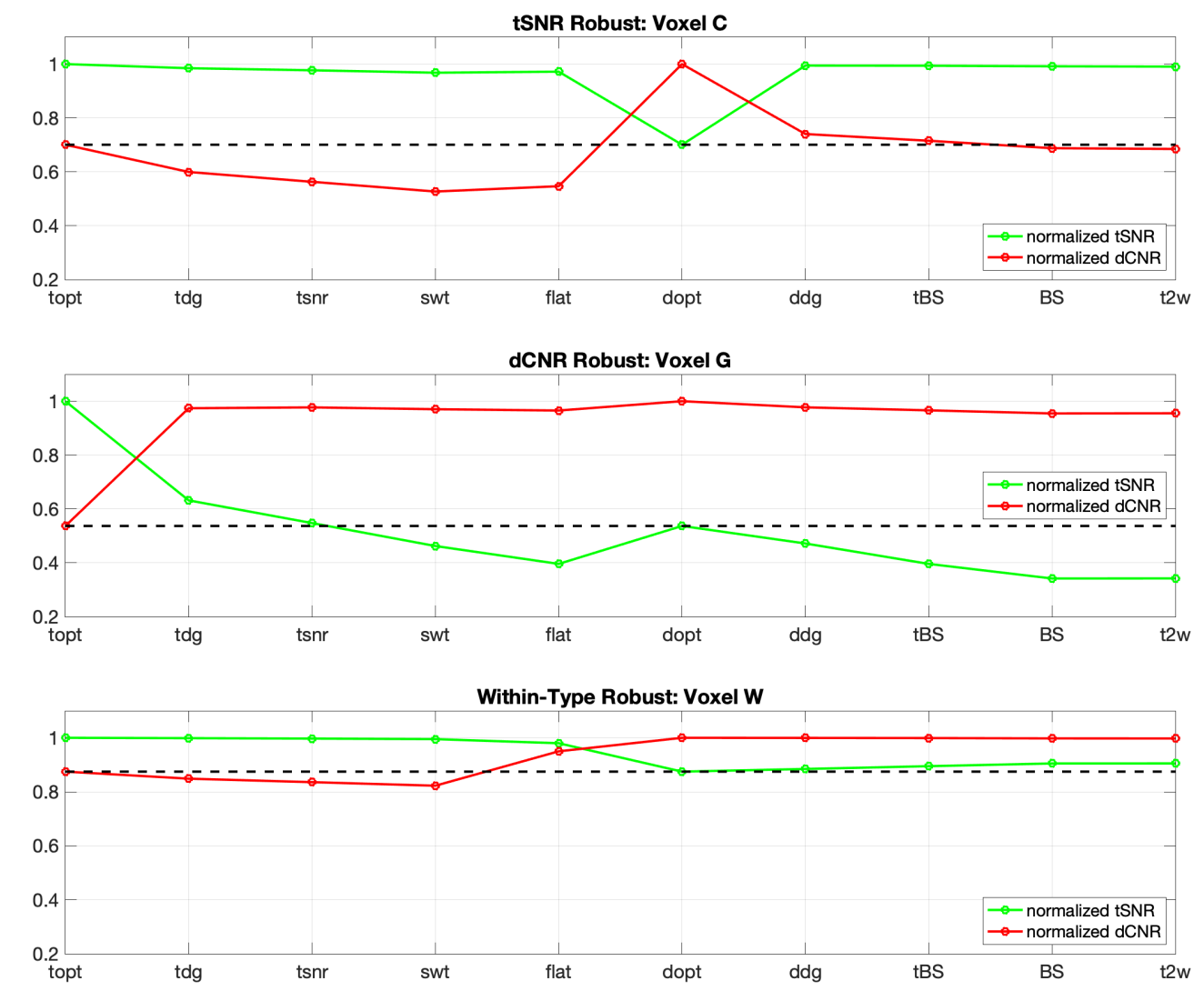

Figure 3: Normalized tSNR and dCNR values plotted versus weight vector variant for the representative voxels indicated in Figure 2 Weight vector variants are labeled with the abbreviations listed in Table 1. The black dotted lines are provided to point out that the normalized tSNR and dCNR values obtained when using dCNR and tSNR optimal weights, respectively, have the same value. 
tSNR Robust Regime
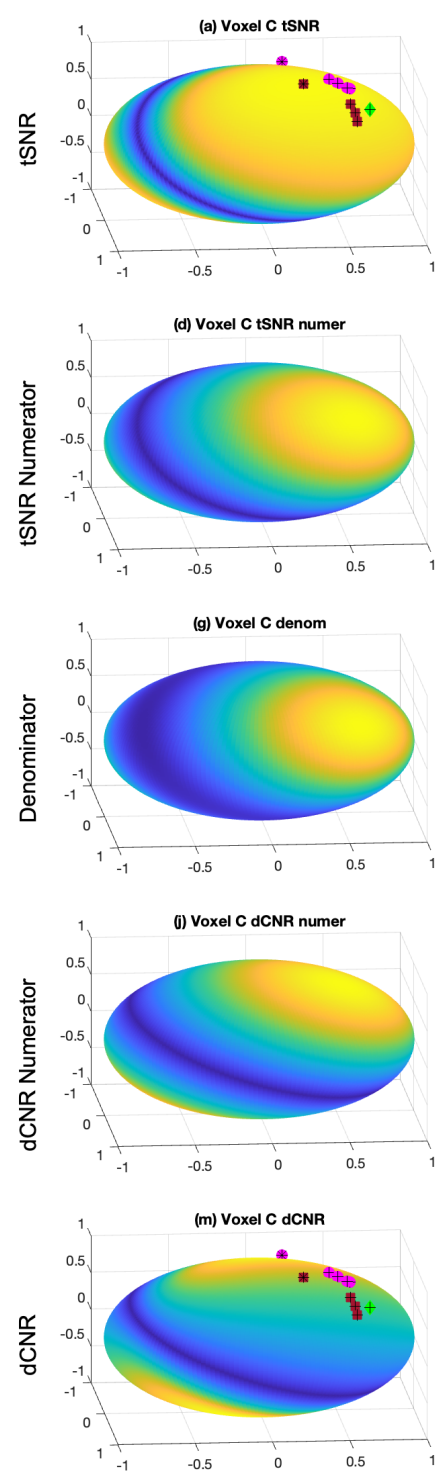

dCNR Robust Regime
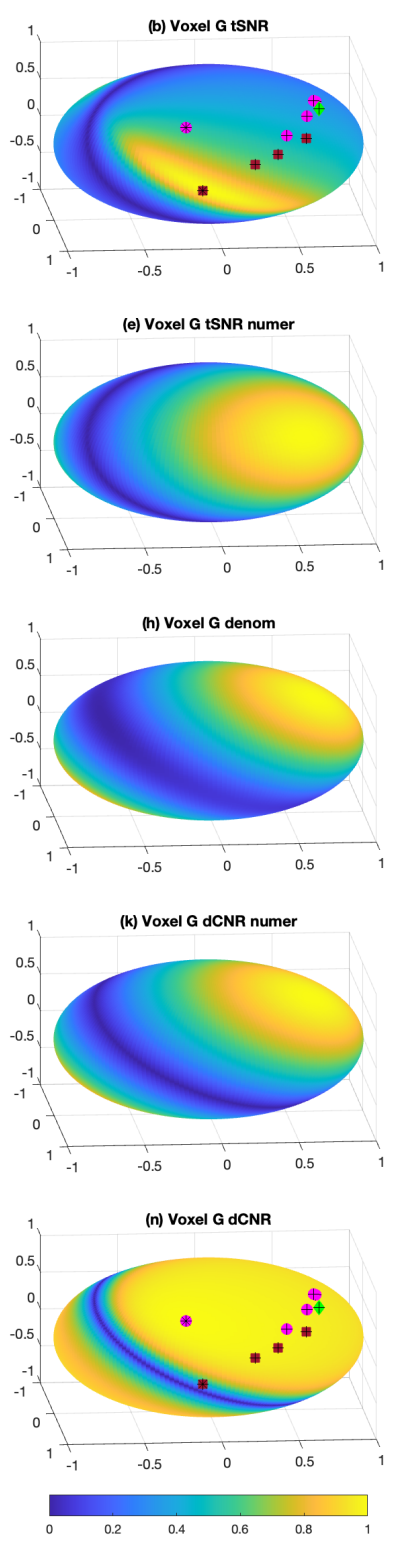

Within-Type Robust Regime
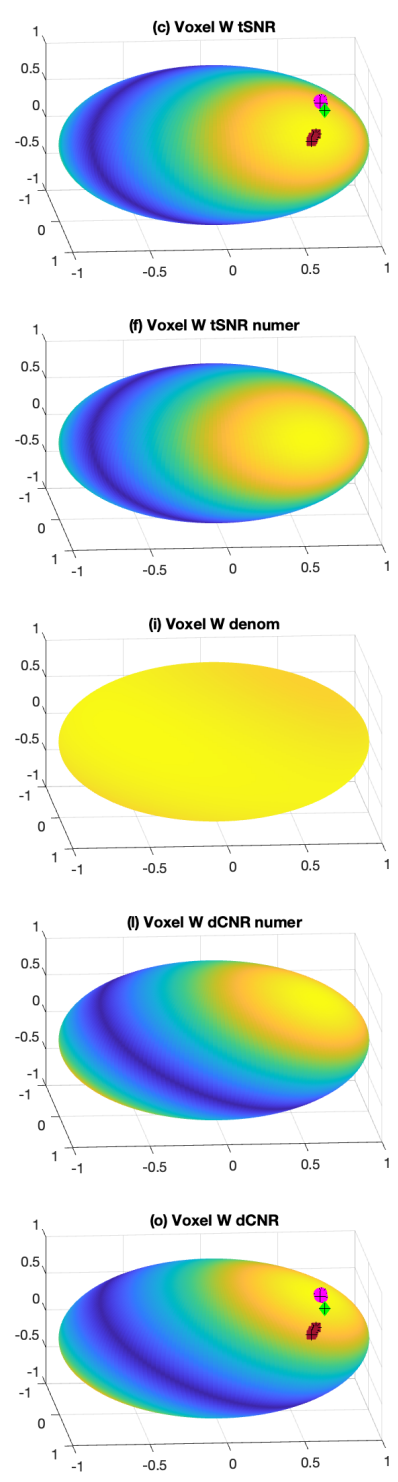

Figure 4: Spherical surface plots showing the weight dependence of (a-c) tSNR, (d-f) tSNR numerator, (g-i) denominator, (j-l) dCNR numerator, and (m-o) dCNR for representative voxels in the tSNR robust regime (voxel C; left column), dCNR robust regime (voxel G; center column), and the within-type robust regime (voxel W; right column). All quantities are shown as absolute values and normalized by their maximum value. The brown squares indicate tSNR weight variants (topt, tdg, tsnr, swt), the magenta circles indicates dCNR weight variants (dopt, ddg, tBS, BS, t2wt), and the green diamonds indicate the flat weight vectors. The optimal tSNR and $\mathrm{dC}_{2} \mathrm{l} R$ weights are indicated with black asterisks while the other variants have black crosses. 